\title{
Validity of local density prescriptions for microscopic calculations of proton nucleus elastic scattering
}

\author{
R. Crespo, ${ }^{*}$ R.C. Johnson, and J.A. Tostevin \\ Department of Physics, University of Surrey, Guildford, Surrey, GU2 5 XH United Kingdom
}

(Received 20 July 1994)

\begin{abstract}
The validity of the local density approximation as applied to the construction of the nucleonnucleus optical potential is studied. A Wentzel-Kramers-Brillouin local (WKB) equivalent potential to the second-order term of the Kerman, McManus, and Thaler (KMT) multiple scattering expansion of the nucleon nucleus optical potential is derived. Assuming that the nucleon-nucleon transition amplitude is on the energy shell, we compare the microscopic KMT optical potential with the approximate potential deduced from the nuclear matter limit by use of the local density approximation. Calculations are presented for the nucleon- ${ }^{16} \mathrm{O}$ system at 135 and $200 \mathrm{MeV}$ incident energies. It is shown that the use of the local density prescription leads to surface peaking of the optical potential. This effect is absent from the second-order term of the optical potential derived microscopically from the KMT approach.
\end{abstract}

PACS number(s): 24.10.Ht, 24.50.+s, 25.40.Cm

\section{INTRODUCTION}

When an incident nucleon scatters from a nucleon in a nucleus rather than in free space there are two basic physical effects which require detailed treatment. One is the action of the Pauli principle, which forbids or blocks certain scattering processes due to the already occupied single-particle states of the finite nucleus. The other is that the incident and struck nucleons interact when each is moving in an external potential field due to the remaining core of target nucleons and which is also responsible for binding the struck particle. The understanding of these effects and thus of the interaction of nucleons with nuclei in terms of the underlying target nucleus structure (wave function) and the elementary free space nucleonnucleon $(N N)$ interaction is of fundamental importance in nuclear physics. A knowledge of this interaction is an input in all scattering and reaction processes, and in particular to high precision photon and electron induced reactions resulting in single- or multiple-nucleon emission.

The clearest microscopic formulation of the nucleonnucleus potential is through multiple scattering expansions, such as those formulated by Kerman, McManus, and Thaler (KMT) [1] and Takeda and Watson [2]. These expand the nucleon-finite-nucleus interaction in orders of an in-medium $N N$ transition amplitude $t_{01}$. The KMT formalism is usually reexpressed in terms of the $N N$ amplitude $t_{01}^{f}\left(\hat{\omega}_{\alpha}\right)$ describing the free scattering of the projectile and struck nucleons, but at appropriate effective $N N$ relative energies $\hat{\omega}_{\alpha}$. These approaches are based from the outset upon a finite nucleus description of the target. The alternative most often used nonrelativistic

\footnotetext{
* On leave from Departamento de Física, Instituto Superior Técnico, Lisboa, Portugal.
}

procedure is to construct a $N N g$-matrix effective interaction appropriate for a pair nucleons interacting in infinite nuclear matter [3]. A local density approximation (LDA) is then used to apply the results to finite nuclei [4-7]. At intermediate energies the KMT and $g$ matrix formalisms are formally identical, to second order in the nucleon-nucleon transition amplitude, as was considered in detail in Ref. [8]. Calculations based on the two approaches, however, disagree quantitatively and it is thus of considerable importance to try to understand the source of such disagreement. This paper attempts to shed some light on this issue, and in particular the role of the LDA, by comparing the microscopic KMT optical potential, evaluated to second order in the $N N$ transition amplitude, with the potential derived from nuclear matter by use of the LDA.

Early theoretical implementations of the KMT and Takeda-Watson multiple scattering expansions suffered in that they assumed only very simple (parametrized) functional forms for the description of the $N N$ scattering amplitude $[9,10]$. Such analyses have advanced rapidly in recent years, due in large part to the work of Redish and Stricker-Bauer [11], who demonstrated the insensitivity of realistic $N N$ amplitudes, both on and off the energy shell, to details of the assumed underlying interaction model. These and other works also made such $N N$ amplitudes readily available, for the best available theoretical $N N$ interaction models, the Paris [12] and Bonn [13] interactions. Very recently, a number of multiple scattering calculations of the nucleon optical potential have been applied to the scattering of protons from doubly closedshell nuclei at intermediate incident energies. Based on the free $N N$ amplitude the nucleon-nucleus interactions thus derived are relatively insensitive to the assumed $N N$ interaction. Additionally, as the on-shell nucleon-nucleon transition amplitude is constrained by experimental $N N$ scattering data, and associated phase shifts, it follows that nucleon-nucleus multiple scattering interactions are 
rather well determined by empirical $N N$ information, at least to the extent that the on-energy-shell behavior is a dominant contribution.

The first-order expression for the KMT optical potential is the expectation value of the free $N N$ amplitude $t_{01}^{f}\left(\hat{\omega}_{\alpha}\right)$ in the target ground state. This term has been the subject of a number of careful calculations using full folding and optimal factorization techniques [14-16]. The key issue here is the appropriate energy $\hat{\omega}_{\alpha}[17]$ at which the $N N$ transition amplitude is to be sampled in evaluating this matrix element. It is now evident $[17,18]$ that a fully consistent treatment of this interaction energy, including the effects of both the struck nucleon binding potential and the spread of energies associated with the center-of-mass motion of the active $N N$ pair, needs to be carried out before definitive conclusions can be drawn about the reliability of such calculations and of the accuracy of the optimal factorization approximation.

The second-order term of the multiple scattering expansion has also been the subject of recent calculations [8]. This term modifies the optical potential of the firstorder term to account for the Pauli blocking effects due to the identity of the target nucleons. The published calculations focused particular attention on the importance of the nonlocalities inherent in the $N N$ amplitude and the intermediate state propagator. Evident from the calculated $S$-matrix elements [8] was that the effects of Pauli blocking resulted in a significant reduction to the nucleon-nucleus absorption, particularly in lower partial waves. Local, phase equivalent interactions to the nonlocal KMT optical potentials [19] confirmed these conclusions and showed that the second-order term reduced the strengths of both the real and the imaginary parts of the central part of the interaction in the nuclear interior. There was also an increase in the strength of the real central interaction at the nuclear surface, and a tendency for surface peaking of the imaginary central part as the incident energy was decreased. These changes at the nuclear surface were, however, smaller than those in the interior and, due to the surface dominance of nucleon-nucleus elastic scattering, the calculated elastic observables did not show large sensitivity to these second-order effects.

Alternatively, $N N$ information can be input into nucleon-nucleus interaction calculations through $N N g$ matrix interactions $g_{01}(\rho)$ appropriate for two nucleons interacting in infinite nuclear matter of density $\rho$ [3] and which take account of Pauli blocking effects within the (infinite) nuclear medium. Several groups have evaluated such $g$ matrices, starting from a variety of free space $N N$ potentials and approximation strategies [4-6]. Inherent in this approach is that a LDA has to be applied to go from nuclear matter to finite nuclei. In essence the $g$ matrix is evaluated for a range of matter densities and then $g_{01}$, at a given radial position $R$ of density $\rho(R)$ in the finite nucleus, is identified with that of infinite nuclear matter of the same density, i.e., $g_{01}(\rho(R))$. To date the validity of such approximations has not been verified by detailed comparisons of finite nucleus results. Moreover, unlike the underlying free $N N$ transition amplitude, the calculated $g_{01}$ exhibit considerable sensitivity to the chosen free space $N N$ potential model [6]. In contrast to the
KMT finite nucleus calculations $[8,19]$ calculated experimental observables, differential cross sections and spin polarizations, at both low and intermediate energies $[4,7]$, are significantly affected by the use of the $g$-matrix interaction, an effect which is attributed to the Pauli blocking effects contained approximately therein. The use of the $g$-matrix interaction does modify the nucleon optical potential in the nuclear surface. The KMT formalism to second order in the free $N N$ amplitude also incorporates Pauli blocking effects in the target medium. It is thus important to try to understand the sources of disagreement between calculations based on these two approaches.

An attempt to understand the different sources of the momentum dependence of the optical potential within the nonrelativistic and relativistic approaches was recently carried out [20]. A study of the validity of the LDA within the relativistic framework was also performed in that work. It was found that the LDA contains the main features of the energy dependence of the optical potential. In the bound state problem the LDA has been found to overestimate the interaction energy for finite systems considerably, particularly for light nuclei [21].

In the present work we first review the KMT optical potential to second order in the underlying $N N$ interaction $t_{01}^{f}\left(\hat{\omega}_{\alpha}\right)$. Further details can be found in Ref. [8]. We also discuss briefly calculations in the nuclear matter limit. Our primary aim is to investigate the accuracy of the LDA in the context of the second-order (Pauli blocking) term of the optical potential. For the purpose of evaluating the optical potential we assume here that the $N N$ amplitude is on the energy shell. In order to clarify the effects of the use of the LDA upon the nuclear matter optical potential, in particular its magnitude and radial form, we derive a WKB local equivalent potential representation, following the formulation of Horiuchi [22]. In this way we are able to compute the local equivalent to the KMT optical potential for comparison with the results of the LDA.

\section{OPTICAL POTENTIAL}

In the KMT formalism the elastic scattering observables are calculated in terms of the nucleon-nucleus $(N A)$ transition amplitude $T^{\mathrm{KMT}}=A /(A-1) T\left(U^{\mathrm{KMT}}\right)$. Here $T\left(U^{\mathrm{KMT}}\right)$ is the transition operator generated from the optical potential $U^{\mathrm{KMT}}$, which is written as an expansion in terms of the $N N$ transition amplitude. If the target ground state wave function is assumed to be a single Slater determinant of occupied single-particle states $|\alpha\rangle$, with single-particle energies $\epsilon_{\alpha}$, then to second order in the $N N$ amplitude the optical potential is $U^{\mathrm{KMT}}=U^{(1)}+U^{(2)}$, where the first-order term $U^{(1)}$ is

$$
U^{(1)}=\frac{A-1}{A} \sum_{\alpha}\left\langle\alpha\left|t_{01}^{f}\left(\hat{\omega}_{\alpha}\right)\right| \alpha\right\rangle
$$

and the second order term $U^{(2)}$ is the sum of two components, $U^{(2)}=U_{\mathrm{I}}^{(2)}+U_{\mathrm{II}}^{(2)}$, where 


$$
\begin{aligned}
& U_{\mathrm{I}}^{(2)}=-\frac{A-1}{A} \sum_{\alpha, \beta}\left\langle\alpha\left|t_{01}^{f}\left(\hat{\omega}_{\beta}\right)\right| \beta\right\rangle \frac{1}{E^{+}-K_{0}+\epsilon_{\alpha}-\epsilon_{\beta}}\left\langle\beta\left|t_{01}^{f}\left(\hat{\omega}_{\alpha}\right)\right| \alpha\right\rangle, \\
& U_{\mathrm{II}}^{(2)}=\frac{A-1}{A^{2}} \sum_{\alpha, \beta}\left\langle\alpha\left|t_{01}^{f}\left(\hat{\omega}_{\alpha}\right)\right| \alpha\right\rangle \frac{1}{E^{+}-K_{0}}\left\langle\beta\left|t_{01}^{f}\left(\hat{\omega}_{\beta}\right)\right| \beta\right\rangle .
\end{aligned}
$$

In these equations the sums in $\alpha$ and $\beta$ run over all occupied states and $t_{01}^{f}\left(\hat{\omega}_{\alpha}\right)$ is the free $N N$ transition operator for the energy parameter $\hat{\omega}_{\alpha}$, the energy of the $N N$ pair in their center-of-mass frame. In the impulse aproximation $\hat{\omega}_{\alpha}$ is thus half of the incident laboratory nucleon energy, $E / 2[15,16]$.

It can be shown $[1,8]$ that the $N A$ transition amplitude satisfies the exact relationship

$$
T^{\mathrm{KMT}}=T(\hat{U})=\frac{A}{A-1} T\left(U^{\mathrm{KMT}}\right),
$$

where the auxilliary potential $\hat{U}$ is

$$
\begin{aligned}
\hat{U}= & \sum_{\alpha}\left\langle\alpha\left|t_{01}^{f}\left(\hat{\omega}_{\alpha}\right)\right| \alpha\right\rangle \\
& -\sum_{\alpha, \beta}\left\langle\alpha\left|t_{01}^{f}\left(\hat{\omega}_{\beta}\right)\right| \beta\right\rangle \frac{1}{\delta_{\alpha \beta}}\left\langle\beta\left|t_{01}^{f}\left(\hat{\omega}_{\alpha}\right)\right| \alpha\right\rangle+\mathcal{O}\left(t^{3}\right),
\end{aligned}
$$

and $\delta_{\alpha \beta}=E^{+}-K_{0}+\epsilon_{\alpha}-\epsilon_{\beta}$.

The meaning of the second term $\hat{U}^{(2)}$ in Eq. (5) is more clear than in Eqs. (2) and (3). Its role is to subtract off contributions to the first-order term from collisions between particles 0 and 1 which produce an intermediate state with particle 1 in an occupied state and which are therefore forbidden by the Pauli principle. The term $U_{\mathrm{II}}^{(2)}$, Eq. (3), arises naturally, however, in the secondorder KMT expansion to account correctly for all contributions from target ground state matrix elements of the $N N$ transition amplitude to the physical scattering amplitude. It was shown in Ref. [8] that at intermediate energies the $g$-matrix and the KMT multiple scattering formalisms are identical to second order in the free $N N$ amplitude, at which energies only the antisymmetrization of the active $N N$ pair and of the $A$ target nucleons need be considered explicitly, as in the KMT implementation.

If we neglect the single-particle energy differences $\epsilon_{\alpha}-$ $\epsilon_{\beta}$ in Eq. (5) [8], the momentum space matrix elements of the interaction $\hat{U}^{(2)}$ can be rewritten

$$
\left\langle\mathbf{k}\left|\hat{U}^{(2)}\right| \mathbf{k}^{\prime}\right\rangle=-\int d \mathbf{k}^{\prime \prime} \beta\left(\omega, \mathbf{k}, \mathbf{k}^{\prime \prime}, \mathbf{k}^{\prime}\right) g\left(k^{\prime \prime}\right) D\left(\mathbf{q}, \mathbf{q}^{\prime}\right),
$$

where $g\left(k^{\prime \prime}\right)$ is the intermediate state propagator (in momentum representation) and $\mathbf{q}=\mathbf{k}-\mathbf{k}^{\prime \prime}$ and $\mathbf{q}^{\prime}=\mathbf{k}^{\prime \prime}-\mathbf{k}^{\prime}$ are the momentum transfers at the two $N N$ vertices. For a target of zero total spin and isospin

$$
\begin{aligned}
\beta\left(\omega, \mathbf{k}, \mathbf{k}^{\prime \prime}, \mathbf{k}^{\prime}\right)= & {\left[\frac{\hbar^{2}}{\mu_{N N}(2 \pi)^{2}}\right]^{2} } \\
& \times\left\{\mathcal{A}^{2}+\mathcal{B}^{2}+2 \mathcal{C}^{2}+\mathcal{D}^{2}+\mathcal{E}^{2}+2 \mathcal{F}^{2}\right\},
\end{aligned}
$$

with $\mu_{N N}$ the $N N$ reduced mass and $\mathcal{A}^{2}-\mathcal{F}^{2}[1]$ are given in terms of the isoscalar and isovector components of the KMT $N N$ amplitudes according to

$$
\mathcal{A}^{2}=\mathcal{A}_{0}^{2}+3 \mathcal{A}_{\tau}^{2} .
$$

We have adopted an abbreviated notation for the squares of the components of the $N N$ amplitude, such that $\mathcal{A}_{0}^{2}=\mathcal{A}_{0}\left(\omega, \mathbf{k}^{\prime}, \mathbf{k}^{\prime \prime}\right) \mathcal{A}_{0}\left(\omega, \mathbf{k}^{\prime \prime}, \mathbf{k}\right)$, and we have made use of a small angle coplanar scattering approximation in treating the unit vectors defining the $N N$ scattering plane. The matrix elements of the second-order optical potential thus depend upon the target structure through the correlation function $D\left(\mathbf{q}, \mathbf{q}^{\prime}\right)$,

$$
D\left(\mathbf{q}, \mathbf{q}^{\prime}\right)=\int d \mathbf{r} \int d \mathbf{r}^{\prime} e^{-i\left(\mathbf{q} \cdot \mathbf{r}+\mathbf{q}^{\prime} \cdot \mathbf{r}^{\prime}\right)} D\left(\mathbf{r}, \mathbf{r}^{\prime}\right) .
$$

Explicitly

$$
D\left(\mathbf{r}, \mathbf{r}^{\prime}\right)=A^{2} \rho(r) \rho\left(r^{\prime}\right)-A(A-1) \rho\left(\mathbf{r}, \mathbf{r}^{\prime}\right),
$$

where $\rho\left(\mathbf{r}, \mathbf{r}^{\prime}\right)$ is the probability density of finding a nucleon at position $\mathbf{r}$ and another at position $\mathbf{r}^{\prime}$, i.e.,

$$
\rho\left(\mathbf{r}, \mathbf{r}^{\prime}\right)=\frac{1}{A(A-1)}\left\langle\phi_{0}\left|\sum_{i \neq j} \delta\left(\mathbf{r}-\mathbf{r}_{i}\right) \delta\left(\mathbf{r}^{\prime}-\mathbf{r}_{j}\right)\right| \phi_{0}\right\rangle
$$

and $\rho(r)$ is the single-particle density, normalized to unity,

$$
\rho(r)=\frac{1}{A}\left\langle\phi_{0}\left|\sum_{i} \delta\left(\mathbf{r}-\mathbf{r}_{i}\right)\right| \phi_{0}\right\rangle .
$$

From Eqs. (10)-(12) it follows that the correlation function is normalized to the number of nucleons $A$,

$$
\int d \mathbf{r} d \mathbf{r}^{\prime} D\left(\mathbf{r}, \mathbf{r}^{\prime}\right)=A
$$

A clear conclusion from our previous studies [8] was that those nonlocalities associated with the $N N$ transition amplitude are not significant in the evaluation of the second-order term of the optical potential. Thus we write [see Eq. (6)]

$$
\left\langle\mathbf{k}^{\prime}\left|\hat{U}^{(2)}\right| \mathbf{k}\right\rangle=-\int d \mathbf{k}^{\prime \prime} t_{01}^{f}(\omega, \mathbf{q}) t_{01}^{f}\left(\omega, \mathbf{q}^{\prime}\right) g\left(k^{\prime \prime}\right) D\left(\mathbf{q}, \mathbf{q}^{\prime}\right)
$$

where $t_{01}^{f}(\omega, \mathbf{q})$ is evaluated on the energy shell. A sum over all components of the scattering amplitude, as in 
Eq. (7), is implicit. The matrix elements of the potential $\hat{U}^{(2)}$ in configuration space are then

$$
\left\langle\mathbf{r}^{\prime}\left|\hat{U}^{(2)}\right| \mathbf{r}\right\rangle=-g\left(\left|\mathbf{r}-\mathbf{r}^{\prime}\right|\right) F\left(\mathbf{r}, \mathbf{r}^{\prime}\right)
$$

with $g\left(\left|\mathbf{r}-\mathbf{r}^{\prime}\right|\right)$ the intermediate state propagator in configuration space. $F\left(\mathbf{r}, \mathbf{r}^{\prime}\right)$ is the folding of the product of the spin-isospin averages of the finite range $N N$ amplitudes with the correlation function,

$F\left(\mathbf{r}, \mathbf{r}^{\prime}\right)=\int d \mathbf{r}_{1} d \mathbf{r}_{2} t_{01}^{f}\left(\omega, \mathbf{r}-\mathbf{r}_{1}\right) t_{01}^{f}\left(\omega, \mathbf{r}^{\prime}-\mathbf{r}_{2}\right) D\left(\mathbf{r}_{1}, \mathbf{r}_{2}\right)$,

where

$$
t_{01}^{f}(\omega, \mathbf{s})=\int d \mathbf{q} t_{01}^{f}(\omega, \mathbf{q}) \exp (i \mathbf{q} \cdot \mathbf{s}) .
$$

Of course in the limit of the zero range approximation to the $N N$ amplitude, the second-order optical potential is simply related to the correlation function, i.e.,

$$
\left\langle\mathbf{r}^{\prime}\left|\hat{U}_{\mathrm{ZR}}^{(2)}\right| \mathbf{r}\right\rangle=-g\left(\left|\mathbf{r}-\mathbf{r}^{\prime}\right|\right)(2 \pi)^{6}\left[t_{01}^{f}(\omega, q=0)\right]^{2} D\left(\mathbf{r}, \mathbf{r}^{\prime}\right) .
$$

To exhibit more clearly the effect of the finite range of the $N N$ interaction, we rewrite in terms of the $N N$ centerof-mass and separation variables $\mathbf{R}_{12}=\left[\mathbf{r}_{1}+\mathbf{r}_{2}\right] / 2$ and $\mathbf{s}_{12}=\mathbf{r}_{2}-\mathbf{r}_{1}$, as

$$
\begin{aligned}
F\left(\mathbf{r}, \mathbf{r}^{\prime}\right)= & \int d \mathbf{R}_{12} d \mathbf{s}_{12} t_{01}^{f}\left(\omega, \mathbf{r}-\mathbf{R}_{12}+\mathbf{s}_{12} / 2\right) \\
& \times t_{01}^{f}\left(\omega, \mathbf{r}^{\prime}-\mathbf{R}_{12}-\mathbf{s}_{12} / 2\right) D\left(\mathbf{R}_{12}, \mathbf{s}_{12}\right) .
\end{aligned}
$$

As a function of $N N$ separation, the correlation function is of longer range than the free $N N$ amplitude. Thus correlation functions that differ in their dependence on this separation are expected to modify the second-order optical potential to some extent. We quantify these changes by considering different correlations in Sec. IV.

To check the validity of the LDA we will consider the function $F$ for nuclear matter of density $\rho$. In this limit the correlation function depends only upon $s_{12}$, and so

$$
\begin{aligned}
F_{\rho}\left(\mathbf{r}-\mathbf{r}^{\prime}\right)= & \int d \mathbf{r}_{1} d \mathbf{r}_{2} t_{01}^{f}\left(\omega, \mathbf{r}-\mathbf{r}_{1}\right) t_{01}^{f}\left(\omega, \mathbf{r}^{\prime}-\mathbf{r}_{2}\right) \\
& \times D\left(\rho, s_{12}\right) .
\end{aligned}
$$

It is clear from Eq. (18) that, in the zero range limit, the accuracy of the LDA is determined only by the extent to which the LDA to the nuclear matter correlation function is a good representation of that of the finite nucleus. Since LDA's are specifically engineered to achieve this reproduction, then in the zero range limit the LDA is expected to be accurate. Application of the LDA to the optical potential, however, takes place at the level of Eq. (20), only after the integrations over the finite ranged $N N$ interaction have been carried out. In those regions where the target density and correlation function vary rapidly with $R_{12}$, with a range comparable with the range of the $N N$ amplitude, the LDA is expected to break down. An assessment of the validity of this approximation for the second-order (Pauli blocking) term of the nucleon optical potential is the main objective of this paper.

\section{LOCAL EQUIVALENT POTENTIAL}

To display the effects of different target correlation functions and of the use of the LDA on the strength and shapes of the derived optical potentials at the nuclear surface, we introduce a local equivalent potential $V^{\mathbf{e q}}(r)$ to the nonlocal second-order KMT optical potential. The wave function for scattering from this potential satisfies the Schrödinger equation

$$
-\frac{\hbar^{2}}{2 \mu_{N A}} \nabla^{2} \Psi_{\mathrm{L}}(\mathbf{r})+V^{\mathrm{eq}}(r) \Psi_{\mathrm{L}}(\mathbf{r})=E \Psi_{\mathrm{L}}(\mathbf{r})
$$

with $\mu_{N A}$ the nucleon target reduced mass. In Ref. [19] we evaluated phase equivalent local potentials which reproduced with high accuracy the elastic partial wave $S$ matrix elements obtained from the full microscopic momentum space calculations. It was found that for energies in excess of $100 \mathrm{MeV}$, the $S$-matrix elements of the second-order KMT potential could be reproduced by a smooth and orbital angular momentum independent local interaction. In the present work we are primarily interested in the study of the effect of the LDA on the second-order term of the KMT potential. To evaluate a local equivalent to this potential term we make use of formalism developed by Horiuchi [22]. This makes use of the WKB approximation which is valid for proton incident energies in the energy region of interest. As described in detail in Sec. IV we are able to obtain an analytic expression for the local equivalent of the second-order term. This allows the effect of assumptions made in the evaluation of the optical potential to be identified very clearly.

We consider first the scattering from an optical potential with local and nonlocal components, written $V(r)+G\left(\mathbf{r}, \mathbf{r}^{\prime}\right)$. The Schrödinger equation thus assumes the form

$$
\begin{aligned}
& -\frac{\hbar^{2}}{2 \mu_{N A}} \nabla^{2} \Psi_{\mathrm{NL}}(\mathbf{r})+V(r) \Psi_{\mathrm{NL}}(\mathbf{r}) \\
& \quad+\int d \mathbf{r}^{\prime} G\left(\mathbf{r}, \mathbf{r}^{\prime}\right) \Psi_{\mathrm{NL}}\left(\mathbf{r}^{\prime}\right)=E \Psi_{\mathrm{NL}}(\mathbf{r})
\end{aligned}
$$

If the nonlocal component satisfies the normal symmetry and rotational invariance requirements, then $G\left(\mathbf{r}, \mathbf{r}^{\prime}\right)$ is a function of the three scalar variables $r^{2},{r^{\prime}}^{2}$, and $\mathbf{r} \cdot \mathbf{r}^{\prime}$, or alternatively of $\left(\mathbf{r}+\mathbf{r}^{\prime}\right)^{2},\left(\mathbf{r}-\mathbf{r}^{\prime}\right)^{2}$, and $\left(\mathbf{r}+\mathbf{r}^{\prime}\right) \cdot\left(\mathbf{r}-\mathbf{r}^{\prime}\right)$.

For the problem of interest, finding a local equivalent to the second-order KMT potential, Eq. (5), we assume $V(r)$ is the local equivalent of the first-order KMT term. In fact we take $V(r)$ to be the local phase equivalent interaction obtained in Ref. [19]. The nonlocal component $G\left(\mathbf{r}, \mathbf{r}^{\prime}\right)$ is then associated with the second-order term 
Eq. (6) in the configuration space representation.

For completeness we describe briefly the local potential prescription of Horiuchi [22]. An equivalent local potential is expressed in terms of the Wigner transform of the nonlocal potential

$$
G^{W}(\mathbf{r}, \mathbf{k})=\int d \mathbf{s} e^{i \mathbf{s} \cdot \mathbf{k}} G(\mathbf{r}-\mathbf{s} / 2, \mathbf{r}+\mathbf{s} / 2)
$$

where rotational invariance implies that $G^{W}(\mathbf{r}, \mathbf{k})=$ $G^{W}\left(r^{2}, k^{2},(\mathbf{r} \cdot \mathbf{k})^{2}\right)$. The local equivalent potential, acting in partial wave $\ell$, is obtained from the Wigner transform after the substitutions

$$
k^{2} \rightarrow \frac{2 \mu_{N A}}{\hbar^{2}}\left[E-V^{\mathrm{eq}}(r)\right]
$$

and

$$
(\mathbf{r} \cdot \mathbf{k})^{2} \rightarrow \frac{2 \mu_{N A} r^{2}}{\hbar^{2}}\left(E-V^{\mathrm{eq}}(r)-\frac{\hbar^{2}(\ell+1 / 2)^{2}}{2 \mu_{N A} r^{2}}\right)
$$

with the result that

$$
V_{\ell}^{\mathrm{eq}}(r)=V(r)+G^{W}\left[r^{2}, \frac{2 \mu_{N A}}{\hbar^{2}}\left[E-V^{\mathrm{eq}}(r)\right], \frac{2 \mu_{N A} r^{2}}{\hbar^{2}}\left(E-V^{\mathrm{eq}}(r)-\frac{\hbar^{2}(\ell+1 / 2)^{2}}{2 \mu_{N A} r^{2}}\right)\right] .
$$

The equivalent potential is in general $\ell$ dependent, which arises from the angular dependence upon the position $\mathbf{r}$ and the momentum $\mathbf{k}$. The radial wave functions generated in the nonlocal $u_{\mathrm{NL}}(r)$ and local $u_{L}(r)$ potentials are related by $[22]$

$$
\begin{aligned}
u_{\mathrm{NL}}(r) & =A(r) u_{L}(r) \\
A(r) & =\left(1+\frac{2 \mu_{N A}}{\hbar^{2}} \frac{\partial G^{W}}{\partial k^{2}}+\frac{2 \mu_{N A} r^{2}}{\hbar^{2}} \frac{\partial G^{W}}{\partial(\mathbf{r} \cdot \mathbf{k})^{2}}\right)^{-1 / 2}
\end{aligned}
$$

\section{TARGET NUCLEUS CORRELATIONS}

\section{A. Single-particle calculations}

Assuming a single determinant (extreme single-particle model) for the target nucleus wave function, the target density is

$$
\rho_{\mathrm{ESP}}(r)=\frac{1}{A} \sum_{\alpha}\left\langle\alpha(1)\left|\delta\left(\mathbf{r}-\mathbf{r}_{1}\right)\right| \alpha(1)\right\rangle
$$

and the two-nucleon correlation function is

$$
\begin{aligned}
D_{\mathrm{ESP}}\left(\mathbf{r}, \mathbf{r}^{\prime}\right) & =\sum_{\alpha \beta}\left\langle\alpha(1) \beta(2)\left|\delta\left(\mathbf{r}-\mathbf{r}_{1}\right) \delta\left(\mathbf{r}^{\prime}-\mathbf{r}_{2}\right)\right| \beta(1) \alpha(2)\right\rangle \\
& =\sum_{\alpha \beta \sigma_{1} \tau_{1} \sigma_{2} \tau_{2}} \alpha^{*}\left(\mathbf{r}, \sigma_{1}, \tau_{1}\right) \beta^{*}\left(\mathbf{r}^{\prime}, \sigma_{2}, \tau_{2}\right) \beta\left(\mathbf{r}, \sigma_{1}, \tau_{1}\right) \alpha\left(\mathbf{r}^{\prime}, \sigma_{2}, \tau_{2}\right) .
\end{aligned}
$$

If we neglect spin-orbit forces, then $\alpha \equiv\left(n_{\alpha}, \ell_{\alpha}, m_{\alpha}\right)$ and

$$
\alpha\left(\mathbf{r}, \sigma_{1}, \tau_{1}\right)=R_{n_{\alpha} \ell_{\alpha}}(r) Y_{\ell_{\alpha} m_{\alpha}}(\hat{r}) \chi_{\sigma_{\alpha}}\left(\sigma_{1}\right) \chi_{\tau_{\alpha}}\left(\tau_{1}\right) .
$$

Upon summing over spin and isospin variables then

$$
D_{\mathrm{ESP}}\left(\mathbf{r}, \mathbf{r}^{\prime}\right)=\left|\varrho\left(\mathbf{r}, \mathbf{r}^{\prime}\right)\right|^{2},
$$

where $\varrho\left(\mathbf{r}, \mathbf{r}^{\prime}\right)$ is the mixed density,

$$
\varrho\left(\mathbf{r}, \mathbf{r}^{\prime}\right)=2 \sum_{n_{\alpha} \ell_{\alpha} m_{\alpha}} R_{n_{\alpha} \ell_{\alpha}}(r) Y_{\ell_{\alpha} m_{\alpha}}^{*}(\hat{r}) R_{n_{\alpha} \ell_{\alpha}}\left(r^{\prime}\right) Y_{\ell_{\alpha} m_{\alpha}}\left(\hat{r}^{\prime}\right) .
$$

For a doubly closed-shell nucleus the single-particle density and correlation function are readily expressed in terms of the single-particle radial wave functions according to

$$
\rho_{\mathrm{SP}}(r)=\frac{1}{A} \frac{1}{\pi} \sum_{n_{\alpha} \ell_{\alpha}} \hat{\ell}_{\alpha}^{2}\left[R_{n_{\alpha} \ell_{\alpha}}(r)\right]^{2}
$$


and

$$
D_{\mathrm{SP}}\left(\mathbf{r}, \mathbf{r}^{\prime}\right)=\frac{1}{4 \pi^{2}} \sum_{n_{\alpha} \ell_{\alpha} n_{\beta} \ell_{\beta}} \hat{\ell}_{\alpha}^{2} \hat{\ell}_{\beta}^{2} R_{n_{\alpha} \ell_{\alpha}}(r) R_{n_{\beta} \ell_{\beta}}(r) R_{n_{\alpha} \ell_{\alpha}}\left(r^{\prime}\right) R_{n_{\beta} \ell_{\beta}}\left(r^{\prime}\right) \sum_{\mathrm{L}}\left(\ell_{\alpha} 0 \ell_{\beta} 0 \mid L 0\right)^{2} P_{L}(\cos \Theta)
$$

where $\hat{\ell}=\sqrt{2 \ell+1}, \Theta$ is the angle between vectors $\mathbf{r}$ and $\mathbf{r}^{\prime}$, and the sums are over occupied states. Assuming a harmonic oscillator (HO) model for the target wave function the $R_{1 \ell}(r)$ states, normalized to unity, are

$$
R_{1 \ell}(r)=\left(\frac{2^{\ell+2}}{\sqrt{\pi} a^{3}(2 \ell+1) ! !}\right)^{1 / 2}\left(\frac{r}{a}\right)^{\ell} \exp \left[-r^{2} /\left(2 a^{2}\right)\right]
$$

with $a=\left(m \omega_{0} / \hbar\right)^{-1 / 2}$ chosen to reproduce the charge density from electron scattering data. For an ${ }^{16} \mathrm{O}$ target,

$$
\rho_{\mathrm{SP}}(r)=\left(\frac{1}{4 \pi^{3 / 2} a^{3}}\right)\left[1+\frac{2}{a^{2}} r^{2}\right] \exp \left(-r^{2} / a^{2}\right)
$$

To compare the correlations from the single-particle and local density approaches it is more convenient to use the coordinates $R$ and $s$, in which

$$
D_{\mathrm{SP}}(R, s)=\frac{4}{\pi^{3} a^{6}}\left\{1+\frac{2}{a^{2}}\left(R^{2}-\frac{s^{2}}{4}\right)\right\}^{2} \exp \left[-2\left(R^{2}+s^{2} / 4\right) / a^{2}\right] .
$$

\section{B. Local density approximations to the correlation function}

We introduce the prescriptions most often used in the application of nuclear matter calculations of the correlation function to finite nuclei. The local density approximation (LDA) to the correlation function considers as a starting point a uniform system of noninteracting fermion-nuclear matter (NM) whose density $\bar{\rho}_{\mathrm{NM}}$ and Fermi momentum $k_{F}$ are related by

$$
k_{F}=\left[3 \pi^{2} \bar{\rho}_{\mathrm{NM}} / 2\right]^{1 / 3} .
$$

The NM correlation function is

$$
D_{\mathrm{NM}}\left(\mathbf{r}, \mathbf{r}^{\prime}\right)=D_{\mathrm{NM}}\left(\bar{\rho}_{\mathrm{NM}}, s\right)=\frac{1}{4}\left[\bar{\rho}_{\mathrm{NM}} G_{\mathrm{FG}}\left(k_{F} s\right)\right]^{2}
$$

where $G_{\mathrm{FG}}(x)$ is the Fermi gas correlation,

$$
G_{\mathrm{FG}}(x)=3 j_{1}(x) / x
$$

and $j_{1}(x)$ is the spherical Bessel function. The finite nucleus [of density $\rho(R)$ ] limit is found using a LDA. The most commonly used of these is the Slater (Sl) approximation in which $\bar{\rho}_{\mathrm{NM}} \rightarrow A \rho(R), k_{F} \rightarrow k_{F}(R)$, to give

$$
\varrho_{\mathrm{Sl}}(\mathbf{R}, \mathbf{s})=\varrho_{\mathrm{Sl}}(R, s)=\frac{1}{2} A \rho(R) G_{\mathrm{FG}}\left(k_{f}(R) s\right)
$$

and hence

$$
k_{F}(R)=\left[3 \pi^{2} A \rho(R) / 2\right]^{1 / 3}
$$

The Slater approximation is, of course, exact only in the limit of an homogeneous infinite system. As was pointed out by Pandariphande [23], the Slater approximation seriously overestimates the mixed density at the nuclear surface. Whereas if the particle density at one of the points $\mathbf{r}, \mathbf{r}^{\prime}$ is zero, the exact mixed density vanishes, and the Slater approximation $\varrho_{\mathrm{S}} \varrho(R, s)$ can nevertheless be finite if the density at the center of mass $\mathbf{R}$ is nonvanishing. For this reason Negele and Vautherin [24] proposed an expansion of the mixed density (DME) in powers of the $N N$ separation about the center-of-mass point. Performing the angular integral over all $N N$ separation directions they obtained, to second order in the gradient of the density, a correction term to the Slater approximation. This term takes into account approximately the departures of the mixed density in the finite nucleus from its value in the nuclear matter.

Campi and Bouyssy (CB) [25] propose an alternative approximation to the mixed density based upon the DME. Essentially they choose an effective average Fermi momentum $\hat{k}_{F}(R)$ so as to cancel the correction term of Negele and Vautherin. For light nuclei, using HO wave functions, this effective Fermi momentum has a simple analytic expression in terms of the $\mathrm{HO}$ parameter. Explicitly, for ${ }^{16} \mathrm{O}[25]$,

$$
\hat{k}_{F}(R)=\left[\frac{5}{3 a^{2}}\left(\frac{9 / 2+3 R^{2} / a^{2}}{1+2 R^{2} / a^{2}}\right)\right]^{1 / 2} .
$$

The mixed density obtained thus has the same simple analytic form as the Slater approximation, but incorporates approximately the finite nucleus deviations.

An alternative prescription for the mixed density was proposed by Pandharipande (Pan) [23],

$$
\varrho_{\mathrm{Pan}}(\mathbf{R}, \mathbf{s})=\frac{1}{2} A \sqrt{\rho(|\mathbf{R}+\mathbf{s} / 2|) \rho(|\mathbf{R}-\mathbf{s} / 2|)} G_{\mathrm{FG}}\left(\tilde{k}_{F}(\mathbf{R}, \mathbf{s}) s\right)
$$


with effective Fermi momentum

$$
\tilde{k}_{F}(\mathbf{R}, \mathbf{s})=\left\{3 \pi^{2} A[\rho(|\mathbf{R}+\mathbf{s} / 2|)+\rho(|\mathbf{R}-\mathbf{s} / 2|)] / 4\right\}^{1 / 3},
$$

which has the proper asymptotic behavior as $\rho(r)$ or $\rho\left(r^{\prime}\right)$ go to zero.

An approximate (app) mixed density with the proper $\rho(r) \rightarrow 0$ asymptotic behavior, but which is more easily handled numerically, is

$$
\varrho_{\mathrm{app}}(\mathbf{R}, \mathbf{s})=\frac{1}{2} A \sqrt{\rho(|\mathbf{R}+\mathbf{s} / 2|) \rho(|\mathbf{R}-\mathbf{s} / 2|)} G_{\mathrm{FG}}\left(k_{F}(R) s\right),
$$

with $k_{F}(R)$ given by Eq. (42). Using HO wave functions, the product of the densities can be written

$$
\rho(|\mathbf{R}+\mathbf{s} / 2|) \rho(|\mathbf{R}-\mathbf{s} / 2|)=\rho_{0}(R, s)+\rho_{2}(R, s) P_{2}(\cos \theta)
$$

with

$$
\begin{aligned}
& \rho_{0}(R, s)=\frac{1}{16 \pi^{3} a^{6}}\left[\left(1+\frac{2}{a^{2}}\left(R^{2}+s^{2} / 4\right)\right)^{2}-\frac{4}{3 a^{4}} R^{2} s^{2}\right] \exp \left[-2\left(R^{2}+s^{2} / 4\right) / a^{2}\right], \\
& \rho_{2}(R, s)=-\frac{1}{6 \pi^{3} a^{10}} R^{2} s^{2} \exp \left[-2\left(R^{2}+s^{2} / 4\right) / a^{2}\right] .
\end{aligned}
$$

The correlation function derived from Eq. (46) is therefore

$$
\begin{aligned}
D_{\text {app }}(\mathbf{R}, \mathbf{s}) & =\frac{1}{4} A^{2} \rho(|\mathbf{R}+\mathbf{s} / 2|) \rho(|\mathbf{R}-\mathbf{s} / 2|)\left[G_{\mathrm{FG}}\left(k_{f}(R) s\right)\right]^{2} \\
& =D_{0}(R, s)+D_{2}(R, s) P_{2}(\cos \theta)
\end{aligned}
$$

The second term accounts for the dependence on the direction of the $N N$ separation vector relative to that of their center of mass.

\section{SECOND-ORDER POTENTIAL}

\section{A. Wigner transform}

The Wigner transform of the second-order potential term is

$$
\begin{aligned}
G^{W}(\mathbf{r}, \mathbf{k}) & =-\int d \mathbf{s} e^{i \mathbf{s} \cdot \mathbf{k}} g(s) F(\mathbf{r}-\mathbf{s} / 2, \mathbf{r}+\mathbf{s} / 2) \\
& =-\int d \mathbf{s} e^{i \mathbf{s} \cdot \mathbf{k}} g(s) \hat{F}(\mathbf{r}, \mathbf{s}) \\
& =\frac{\mu_{N A}}{2 \pi \hbar^{2}} \int d s s e^{i k_{0} s} \int d \Omega_{s} e^{i \mathbf{s} \cdot \mathbf{k}} \hat{F}(\mathbf{r}, \mathbf{s}),
\end{aligned}
$$

where $\hat{F}(\mathbf{r}, \mathbf{s})$ is precisely the function $F$ but expressed in terms of the $N N$ center-of-mass and separation variables. The function $\hat{F}(\mathbf{r}, \mathbf{s})$ can be written $(\mu=\hat{s} \cdot \hat{r})$

$$
\begin{aligned}
\hat{F}(\mathbf{r}, \mathbf{s}) & =\hat{F}\left(r, s, \mu^{2}\right) \\
& =\hat{F}_{0}(r, s)+\hat{F}_{2}(r, s) P_{2}(\mu) \\
& =\hat{F}_{0}^{\prime}(r, s)+\hat{F}_{2}^{\prime}(r, s)(\hat{s} \cdot \hat{r})^{2},
\end{aligned}
$$

with

$$
\begin{aligned}
& \hat{F}_{0}^{\prime}(r, s)=\hat{F}_{0}(r, s)-\hat{F}_{2}(r, s) / 2, \\
& \hat{F}_{2}^{\prime}(r, s)=3 \hat{F}_{2}(r, s) / 2 .
\end{aligned}
$$

We proceed by evaluating the angular integral appearing in the Wigner transform Eq. (50), with the result that

$$
\begin{aligned}
G^{W}(\mathbf{r}, \mathbf{k}) & =G^{W}\left(r^{2}, k^{2},(\mathbf{r} \cdot \mathbf{k})^{2}\right) \\
& =G_{0}^{W}\left(r^{2}, k^{2}\right)+G_{2}^{W}\left(r^{2}, k^{2},(\mathbf{r} \cdot \mathbf{k})^{2}\right)
\end{aligned}
$$

with

$$
\begin{aligned}
G_{0}^{W}\left(r^{2}, k^{2}\right) & =\mathcal{I}_{0}(k, r), \\
G_{2}^{W}\left(r^{2}, k^{2},(\mathbf{r} \cdot \mathbf{k})^{2}\right) & =\mathcal{I}_{2}(k, r)(\hat{r} \cdot \hat{k})^{2},
\end{aligned}
$$

and where

$$
\mathcal{I}_{\lambda}(k, r)=i^{\lambda} \frac{2 \mu_{N A}}{\hbar^{2}} \int d s s j_{\lambda}(k s) e^{i k_{0} s} \hat{F}_{\lambda}^{\prime}(r, s) .
$$

The $\lambda=0$ component of the second-order potential is therefore given by

$$
U_{\lambda=0}^{(2)}(r)=\frac{2 \mu_{N A}}{\hbar^{2}} \int d s s j_{0}(k s) e^{i k_{0} s} \hat{F}_{0}^{\prime}(r, s)
$$

and, for a spherical nucleus, using HO target wave functions, the second-order term of the KMT potential can be represented by a local $\ell$-independent equivalent potential. This is the expression calculated numerically here.

We note that in the high-energy limit, assuming the effective momentum $k$ in the Wigner transform can be fixed to the incident momentum $k \sim k_{0}$, as is appropriate at high energies,

$$
j_{0}\left(k_{0} s\right) e^{i k_{0} s} \sim \frac{i}{2 k_{0} s},
$$


this expression may be simplified further to give

$$
U_{\lambda=0}^{(2)}(r) \sim \frac{i \mu_{N A}}{\hbar^{2} k_{0}} \int d s \hat{F}_{0}^{\prime}(r, s),
$$

where the explicit $1 / k_{0}$ factor will reduce the Pauli blocking effects with increasing incident energy.

\section{B. Local potential representation}

To arrive at an analytic expression for the Wigner transform we now assume that the $N N$ amplitude is on the energy shell. Thus, in momentum space, Eq. (7) becomes $\beta\left(\omega, q, q^{\prime}\right)$ with $q$ and $q^{\prime}$ the $N N$ momentum transfers and where $\mathcal{A}_{0}^{2}=A_{0}(\omega, q) A_{0}\left(\omega, q^{\prime}\right)$, etc. In the present work we parametrize $\beta\left(\omega, q, q^{\prime}\right)$ with a Gaussian momentum transfer dependence as

$$
\beta\left(\omega, q, q^{\prime}\right)=\beta_{0}(\omega) \exp \left(-\tilde{c}\left[q^{2}+q^{\prime 2}\right]\right),
$$

where $\beta_{0}(\omega)$ is the $q, q^{\prime} \rightarrow 0$ limit of $\beta\left(\omega, q, q^{\prime}\right)$. Thus, in the configuration space integrals we replace

$$
t_{01}^{f}(\omega, s) t_{01}^{f}\left(\omega, s^{\prime}\right)=\hat{\beta}_{0}(\omega) \exp \left(-c\left[s^{2}+s^{\prime 2}\right]\right),
$$

where $c=1 / 4 \tilde{c}$ and $\hat{\beta}_{0}(\omega)=[4 c \pi]^{3} \beta_{0}(\omega)$. Thus the $N N$ dynamics will determine the strength of the secondorder potential, essentially through $\hat{\beta}_{0}(\omega)$. The choice of a Gaussian parametrization is one only of simplicity and implies an approximate description of the configuration space $N N$ form factor. Such detailed considerations of radial form are not relevant, however, to the main purpose of the present work. The choice of parameters in the parametrization is discussed in the following section.

With the adopted parametrization the functions $\hat{F}_{\lambda}(r, s),(\lambda=0,2)$ for the microscopic KMT interaction are now expressed as double radial integrals, with the target correlation function, as

$$
\begin{aligned}
\hat{F}_{\lambda}(r, s)= & \hat{\beta}_{0}(\omega) e^{-c\left[4 r^{2}+s^{2}\right] / 2} \int R_{12}^{2} d R_{12} \int s_{12}^{2} d s_{12} \\
& \times D_{\lambda}\left(R_{12}, s_{12}\right) \mathcal{R}_{\lambda}\left(r, s, R_{12}, s_{12}\right)
\end{aligned}
$$

Here $D_{\lambda}\left(R_{12}, s_{12}\right)$ can be replaced by either the finite nucleus (single-particle model) correlation or one of the LD approximations to it, as discussed in Sec. IV. Explicit formulas for the $\hat{F}_{\lambda}(r, s)$ are collected in the Appendix. The $\lambda=2$ component arises only when we consider the approximate local mixed density denoted "app" in Sec. IV.

Alternatively, in the case of the LDA for the optical potential deduced from the nuclear matter limit, the density-dependent optical potential, we obtain

$$
\begin{aligned}
\hat{F}^{\mathrm{LDA}}(r, s)= & \hat{F}_{\rho(r)}(s) \\
= & \hat{\beta}_{0}(\omega) e^{-c s^{2} / 2} \int d s_{12} s_{12}^{2} \mathcal{R}^{\mathrm{LDA}}\left(s, s_{12}\right) \\
& \times D\left(\rho(r), s_{12}\right)
\end{aligned}
$$

and hence

$$
U^{\mathrm{LDA}}(r)=\frac{2 \mu_{N A}}{\hbar^{2}} \int d s s j_{0}(k s) e^{i k_{0} s} \hat{F}^{\mathrm{LDA}}(r, s)
$$

where the fact that this is the second-order term is understood implicitly. The explicit formula for $\hat{F}^{\mathrm{LDA}}(r, s)$ is included in the Appendix.

The self-consistent optical potentials are calculated by replacing, initially, the momentum $k$ in the Wigner transform by the incident momentum $k_{0}$, such that the lowestorder estimate of the local second-order potential term is

$$
U_{\lambda=0}^{(2)}(r)=\frac{2 \mu_{N A}}{\hbar^{2}} \int d s s j_{0}\left(k_{0} s\right) e^{i k_{0} s} \hat{F}_{0}^{\prime}(r, s) .
$$

Given the local equivalent $V(r)$ to the first-order KMT potential and the estimated $U_{\lambda=0}^{(2)}(r)$, Eq. (57) or (64) can now be iterated to convergence with $k$ calculated at each iteration from Eq. (24) with $V^{\text {eq }}(r)=V(r)+U_{\lambda=0}^{(2)}(r)$. Similarly, in the case of the use of $D_{\text {app }}(\mathbf{R}, \mathbf{s})$, where the $\lambda=2$ component is nonvanishing and the resulting optical potential is $\ell$ dependent, Eq. (53) is iterated to convergence with $k$ calculated from Eq. (24) with $V^{\mathbf{e q}}(r)=V(r)+U_{\lambda=0}^{(2)}(r)+\mathcal{I}_{\lambda=2}(k, r)(\hat{r} \cdot \hat{k})^{2}$ and $(\mathbf{r} \cdot \mathbf{k})^{2}$ given by Eq. (25).

\section{NUMERICAL RESULTS}

\section{A. Nucleon-nucleon amplitudes}

In previous studies of the second-order KMT optical potential $[8,19]$ the required free $N N$ amplitudes were calculated exactly, both on and off the energy shell, from the Paris potential $[12,26]$. In the present work the $N N$ amplitudes are assumed on the energy shell. A detailed evaluation of the second-order term of the KMT potential was presented in [8]. Our aim here is to provide insight into the validity of local density approximations to the second-order (Pauli blocking) term of the optical potential and we have therefore assumed the simplified Gaussian parametrized form of the $\beta$ function, Eq. (7), as a function of the $N N$ energy and momentum transfer, and given by Eq. (60).

In using Eq. (60) the overall normalization $\beta_{0}(\omega)$ was assigned the value calculated from the Paris $N N$ potential, Eq. (7), at zero momentum transfers, $q=q^{\prime}=0$. The Gaussian range parameter $\tilde{c}$, at 135 and $200 \mathrm{MeV}$ incident energies, was chosen such that the imaginary part of the calculated local potential to the second term was consistent with that obtained from the fully microscopic second-order calculations of Ref. [19]. We obtained the values $\tilde{c}=0.95 \mathrm{fm}^{2}$ and $\tilde{c}=0.75 \mathrm{fm}^{2}$ at 135 and $200 \mathrm{MeV}$, respectively. A comparison of the calculated $\beta\left(\omega, q, q^{\prime}\right)$ with these parameters, dashed curves, with the exact Paris potential $\beta$ function Eq. (7), solid curves, for the diagonal ( $q=q^{\prime}$ ) elements is presented in Fig. 1. We note that the Gaussian parametrization does provide a reasonable description of the imaginary parts of the relevant $N N$ form factor for $q<1.5 \mathrm{fm}^{-1}$, at both 135 and $200 \mathrm{MeV}$, and also follows the main trend of the real parts of the amplitude. 

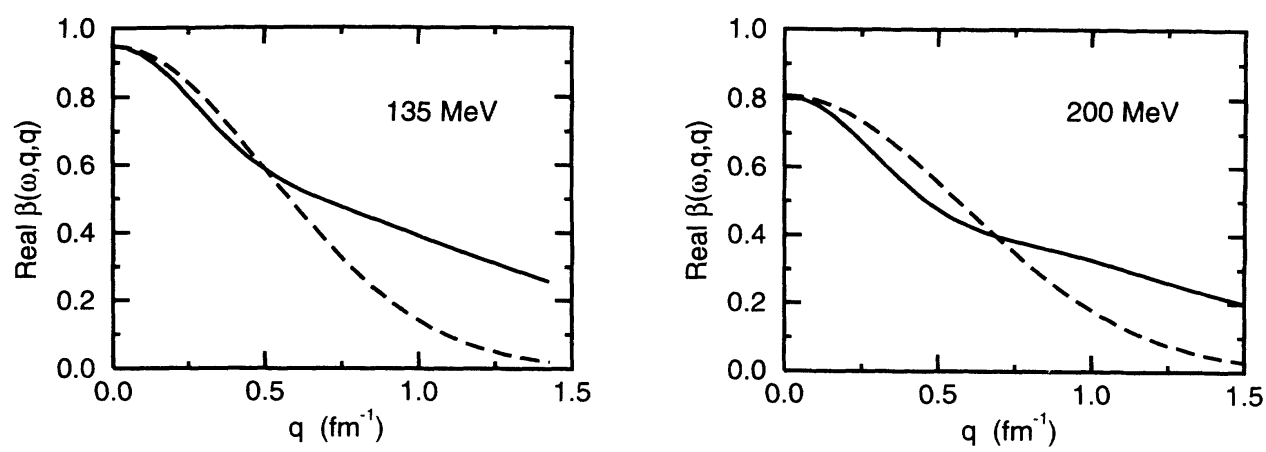

FIG. 1. Comparison of the calculated $\beta(\omega, q, q)$ with $\tilde{c}=0.95 \mathrm{fm}^{2}(135 \mathrm{MeV})$ and $\tilde{c}=0.75 \mathrm{fm}^{2}(200 \mathrm{MeV})$
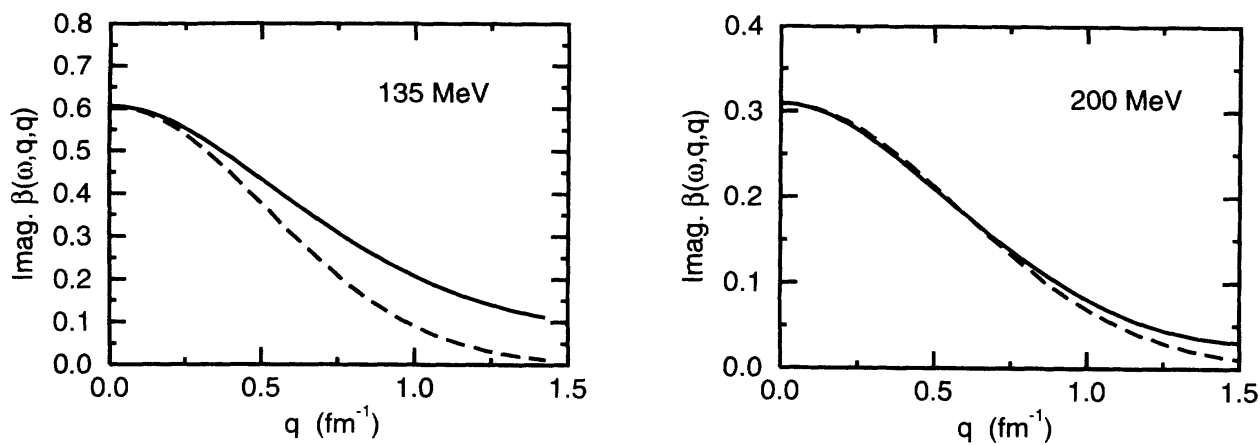

(dashed curves) with the exact $\beta(\omega, q, q)$ derived from the Paris potential (solid curves).

\section{B. Correlation functions}

In this subsection we consider briefly the extreme single-particle model correlation function for an ${ }^{16} \mathrm{O}$ target and the local density approximations to it. For consistency we use the harmonic oscillator (HO) description of the target single-particle functions in calculating both the single-particle, and the LDA's to the correlation function. The oscillator range parameter $a=1.77 \mathrm{fm}$ was taken from
Donnelly and Walker [27].

Figure 2 shows the correlation function, in the form of an effective $N N$ correlation $\left[G_{\text {eff }}(\mathbf{R}, \mathbf{s})\right]^{2}=$ $4 D(\mathbf{R}, \mathbf{s}) /[A \rho(R)]^{2}$, as a function of the $N N$ separation $s$ for several values of $N N$ center-of-mass position $R$. In the limit of nuclear matter the presented function thus reduces to the square of the Fermi gas correlation $\left[G_{\mathrm{FG}}\left(k_{F} s\right)\right]^{2}$, of Eq. (40). The curves show the single-particle model results (solid line) and the CB
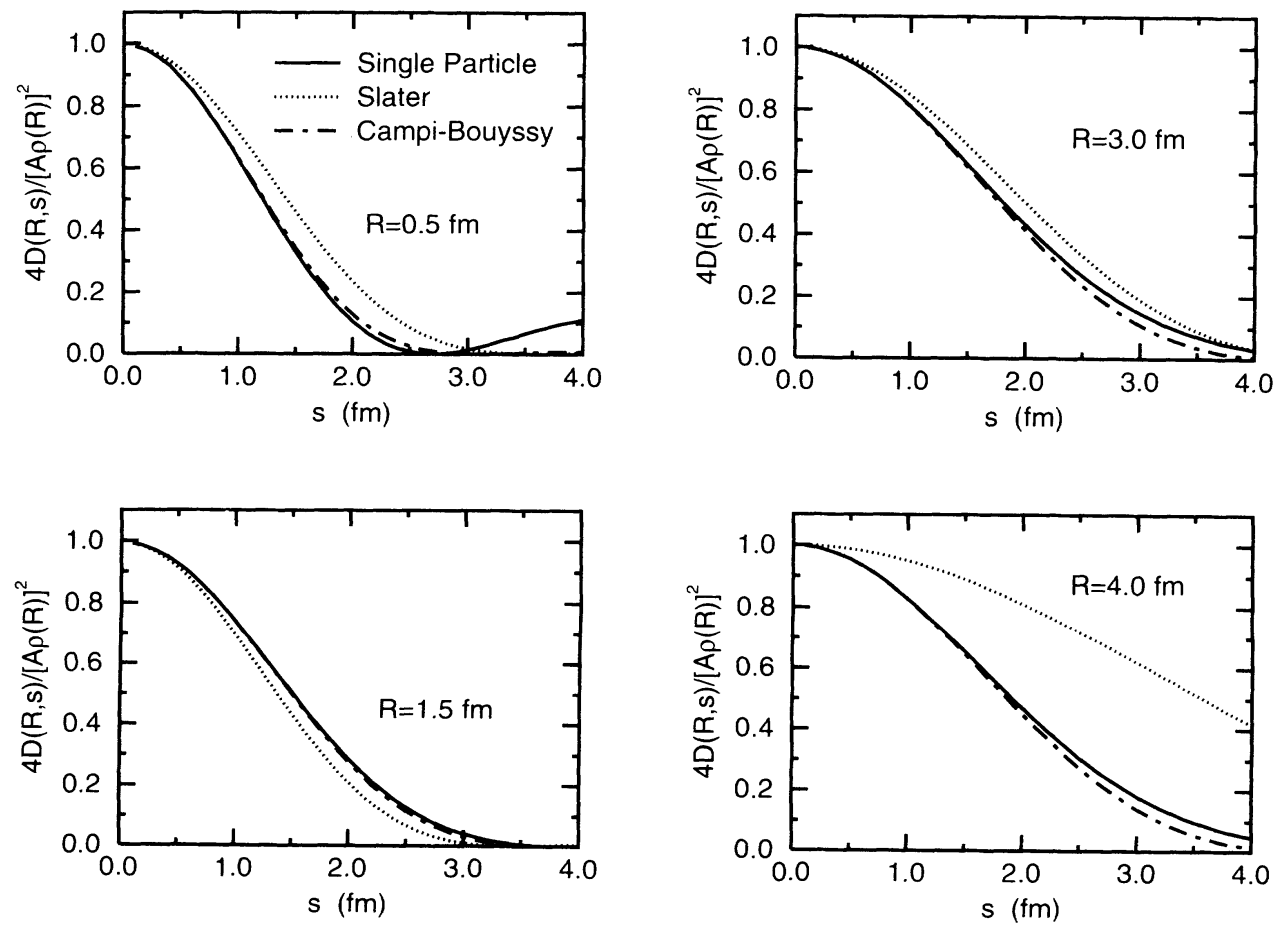

FIG. 2. Calculated correlation function, in the form $4 D(\mathbf{R}, \mathrm{s}) /[A \rho(R)]^{2}$, as a function of the $N N$ separation $s$ for several values of $N N$ center-of-mass position $R$. The curves show the single-particle results (solid line) and the CB (dot-dashed line) and Slater (dotted line) approximations to it. 
(dot-dashed line) and Slater (dotted line) approximations to it. Whereas the effective Fermi momentum of the Slater approximation decays rapidy to zero, as $\rho(R)^{1 / 3} \sim$ $R^{2 / 3} \exp \left[-R^{2} /\left(3 a^{2}\right)\right]$, the CB expression $\hat{k}_{F}(R)$ decays only slowly, and to a constant value in the case of $\mathrm{HO}$ single-particle functions. As would be expected from this behavior and the form of $G_{\mathrm{FG}}$, the Slater approximation to the correlation function at the nuclear surface has a longer tail as a function of the $N N$ separation. In the case of the description of Pandariphande (Pan) and of the approximate (app) description, Fig. 3, the results are shown as open and solid circles and as solid and dashed lines and for $\mathbf{R}$ perpendicular to $\mathbf{s}$ and $\mathbf{R}$ parallel to $\mathbf{s}$, respectively. The approximate correlation function $D_{\text {app }}$ also gives a very reasonable description of the Pandariphande (Pan) prescription. The Campi-Bouyssy local density approximation gives a very reliable representation of the finite nucleus single-particle correlation function.

\section{Optical potential}

Using the correlation functions above, the local equivalent to the second-order term of the optical potential can be calculated from both the finite nucleus viewpoint and, using the LDA, from the nuclear matter limit. All calculations presented here relate to nucleon- ${ }^{16} \mathrm{O}$ scattering at 135 and $200 \mathrm{MeV}$.

\section{Microscopic optical potential}

Figure 4 shows the real and imaginary parts of the local equivalent to the second-order term of the micro- scopic optical potential $U_{0}^{(2)}(r)$, Eq. (57), at $135 \mathrm{MeV}$. The curves show the single-particle (solid line), CB (dotdashed line), and Slater (dotted line) approximations to the Pauli blocking term where the $\hat{F}_{0}(r, s)$ are calculated from Eq. (62). The Campi-Bouyssy LDA to the correlation function gives a good representation of the singleparticle optical potential, as was expected given the results presented in Fig. 2. The potential derived from the Slater approximation decays more slowly than the corresponding single-particle-based potential. Nevertheless, it gives qualitatively the same results, despite giving a poor description of the correlation function at the nuclear surface, since only the short range behavior of the correlation function is sampled in the evaluation of the optical potential. The figure also shows the corresponding optical potential calculations at $200 \mathrm{MeV}$ with qualitatively similar results.

In Fig. 5 we compare the calculated local second-order terms $U_{0}^{(2)}(r)$ at 135 and $200 \mathrm{MeV}$ of the present work, and derived from the Wigner transform, with those obtained by inversion of the $S$-matrix elements of the complete microscopic calculations described in Refs. $[8,19]$. Both the real and imaginary parts of the second-order term of the optical potential from the Wigner transform approach provide a quantitative representation of the phase equivalent local potentials of [19], particularly in the nuclear surface of particular interest for the discussion of the LDA presented here. The main features of the small real part of the optical potential are correctly generated, but the interior contribution is not accurately reproduced. These results give us confidence in the ability of the model derived to determine the essential qualitative and quantitative features of the use of the LDA for the Pauli blocking term of the microscopic optical
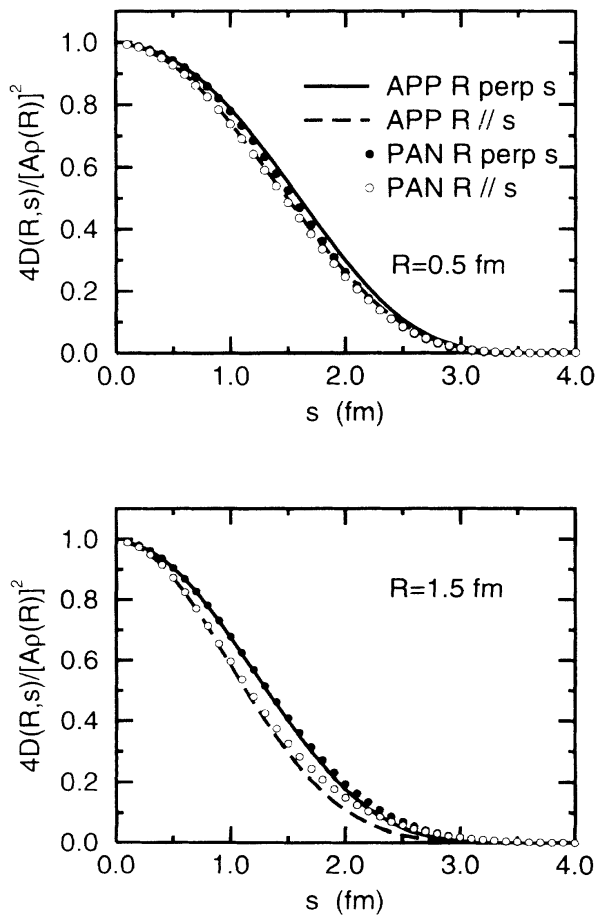
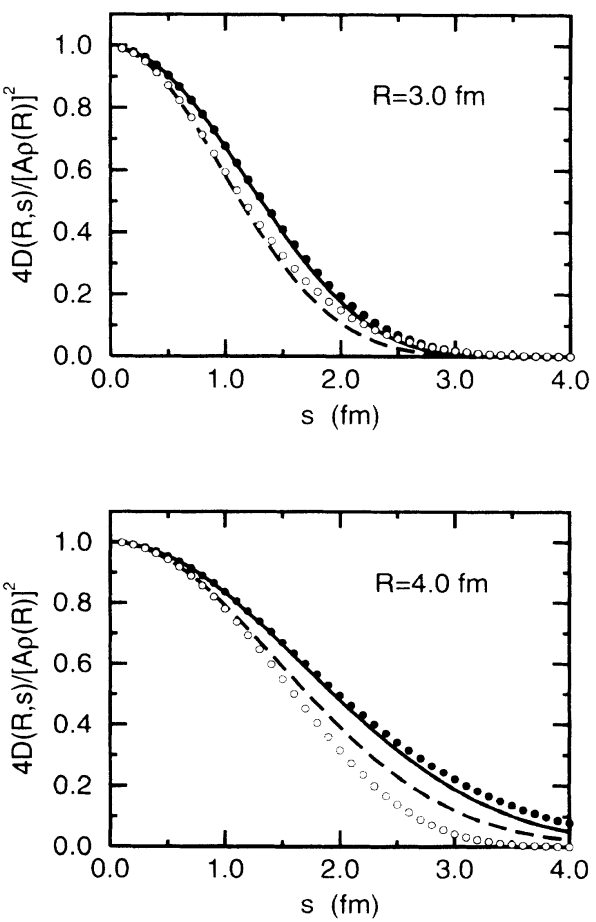

FIG. 3. As for Fig. 2 for the Pandariphande (Pan) and approximate (app) descriptions of the correlation function. The circles (PAN) and the curves (app) correspond to $\mathbf{R}$ perpendicular to $\mathbf{s}$ and $\mathbf{R}$ parallel to $\mathbf{s}$, respectively. 

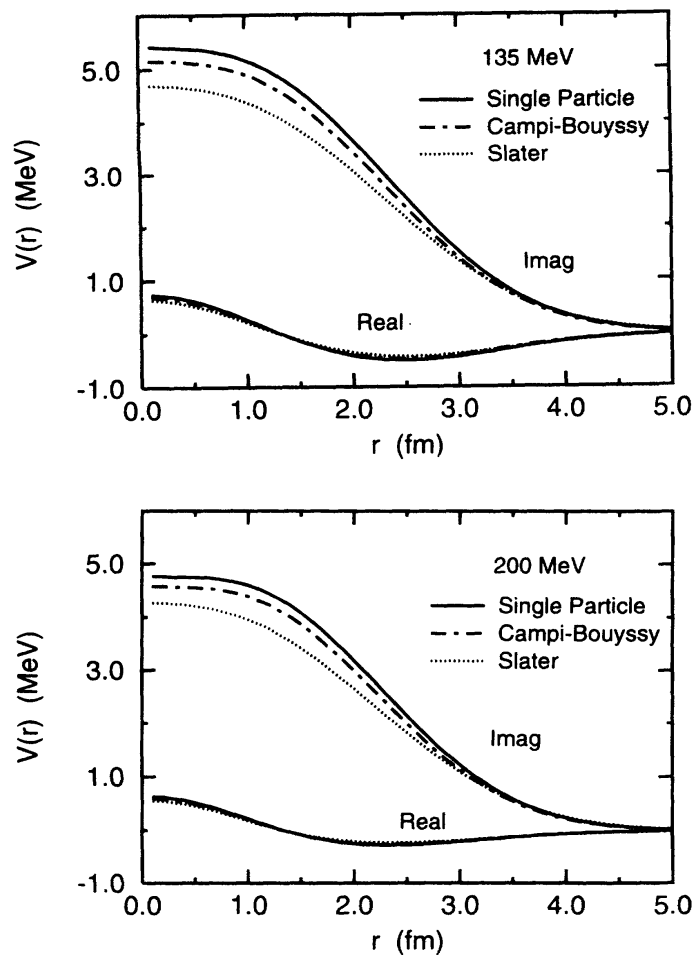

FIG. 4. Calculated real and imaginary parts of the local equivalent to the second-order term of the microscopic optical potential at $135 \mathrm{MeV}$ (upper figure) and $200 \mathrm{MeV}$ (lower figure). The curves show the single-particle (solid line), and approximate CB (dot-dashed line) and Slater (dotted line) results.
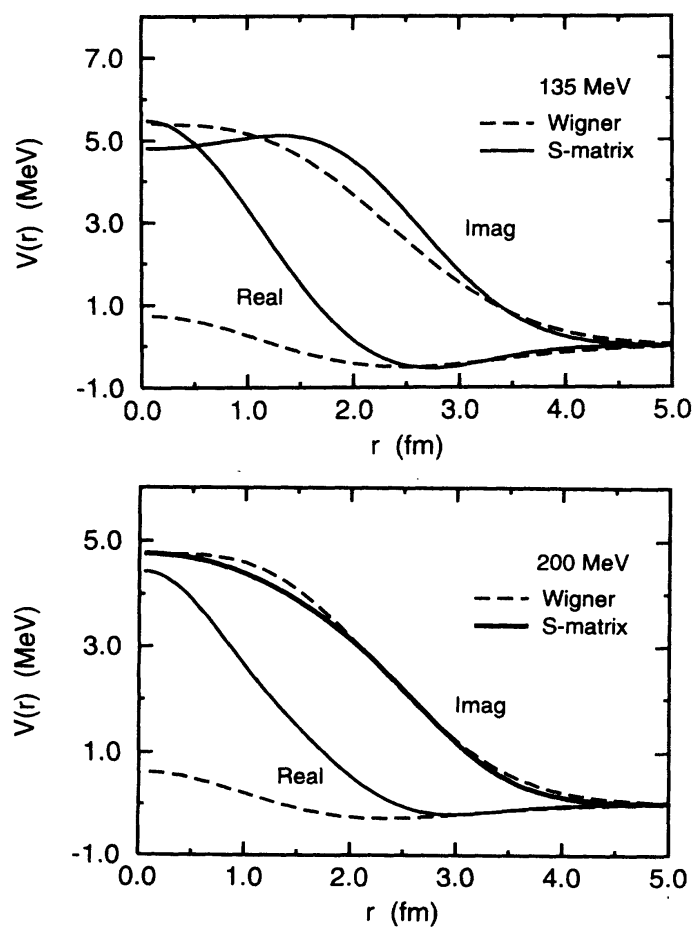

FIG. 5. Comparison of the approximate local second-order term at 135 and $200 \mathrm{MeV}$ as described in the text with that obtained from the complete microscopic calculations of Refs. $[8,19]$. potential.

Figure 6 presents the real and imaginary parts of the second-order term of the optical potential derived from the approximate correlation function, denoted $D_{\mathrm{app}}$ earlier, and which was introduced to approximate (see Fig. 3) the Pandharipande correlation function, but in a rather simpler numerical form. As was noted earlier, the residual dependence in these descriptions of the correlation function, upon the directions of the $N N$ center-ofmass and separation vectors, leads to the derived optical potentials being partial wave $(\ell)$ dependent. The figure presents the potentials calculated in the $\ell=0,10,20$, and 30 partial waves. As follows from the figure the $\ell$ dependence is relatively weak over the range of relevant orbital angular momenta and the underlying interaction is quantitatively very similar to that of the earlier used correlation functions.

\section{Local density approximation calculations}

In Fig. 7 we compare these microscopic second-order terms of the optical potential at $135 \mathrm{MeV}$, derived from Eq. (62), with the density-dependent (DD) approximation to the optical potential calculated from $\hat{F}^{\text {LDA }}(r, s)$ of Eq. (63). The dot-dashed and dotted curves are the same as those in Fig. 4 and result from using the CB and Slater approximations to the correlation function in
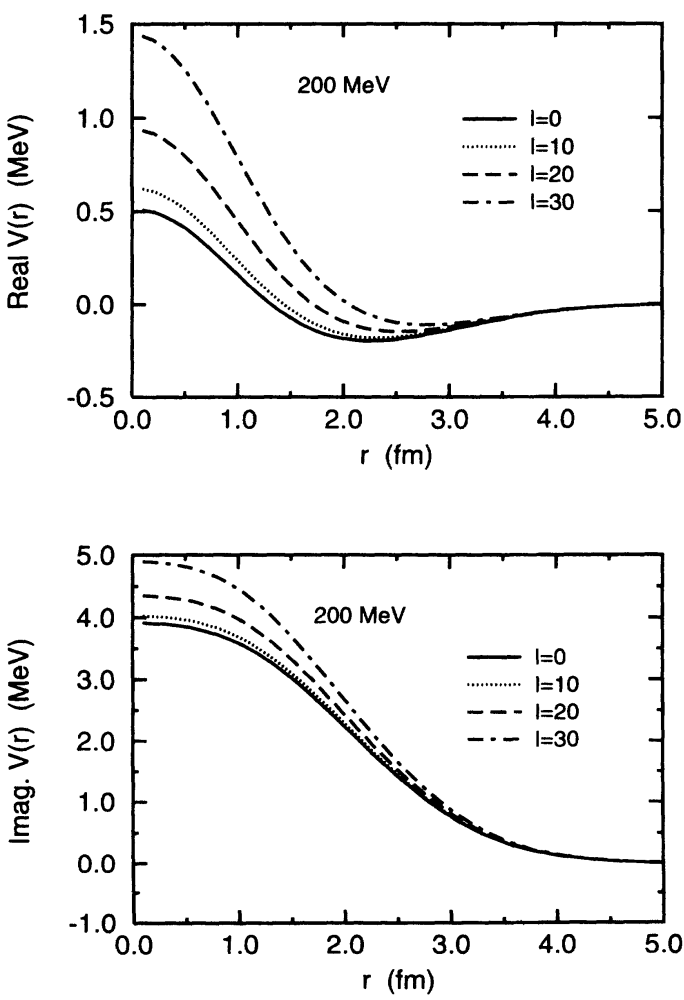

FIG. 6. Calculated real and imaginary parts of the $\ell$-dependent second-order term of the potential, from the approximate correlation function $D_{\text {app }}$, at $200 \mathrm{MeV}$ in the $\ell=0$, 10,20 , and 30 partial waves. 
Eq. (62). The solid and long-dashed curves show the potentials derived from the nuclear matter limit by the use of the CB and Slater correlation functions in $\hat{F}^{\mathrm{LDA}}(r, s)$. Figure 8 shows the corresponding curves at $200 \mathrm{MeV}$. It is clear from the figures that the LDA to the nuclear matter optical potential results in a significantly more surface peaked potential than is obtained microscopically from the finite nucleus correlation function through Eq. (62).

Figure 9 shows the zero range (of the $N N$ amplitude) limit of the second-order term of the optical potential derived from the single-particle correlation function (solid line), and the density dependent optical potentials derived from CB (dot-dashed line) and Slater (dotted line) correlation functions at 135 and $200 \mathrm{MeV}$. Comparison with Figs. 6 and 7 shows that, as expected, the zero range approximation leads to a considerable overestimation of the optical potential, as was obtained in the work of Ref. [8]. The similarity between all calculations in this case reflects the fact that the LDA is valid in the limit of the zero range approximation, the deviations reflecting only the effects of differences of the Slater and CB approximations to the correlation function in Eq. (18).

\section{Elastic scattering observables}

In all calculations in this section the approximate methods of this paper are used to obtain the local secondorder term of the optical potential. These potentials are then added to the local representation of the first-order term of the KMT potential obtained in [19], including its spin dependence, and the observables calculated. The Coulomb interaction is not included and is of no relevance to the present discussion.

To clarify the significance of the use of approximate

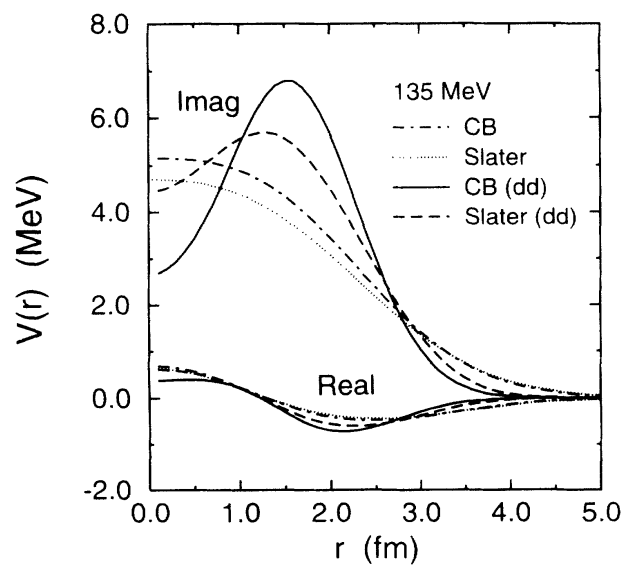

FIG. 7. Comparison of the microscopic second-order term of the optical potential at $135 \mathrm{MeV}$ with the density-dependent (DD) approximation to the optical potential as described in the text. The dot-dashed and dotted curves result from using the $\mathrm{CB}$ and Slater approximations to the correlation function as in Fig. 4. The solid and long-dashed curves show the corresonding density-dependent (DD) potentials derived from the nuclear matter limit.

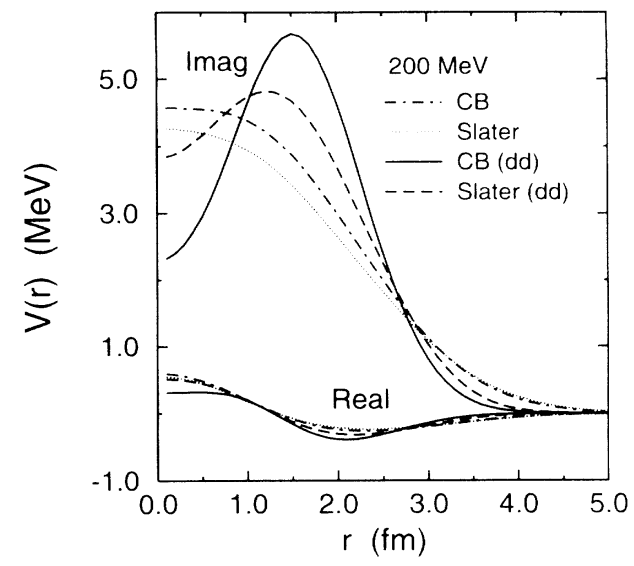

FIG. 8. As for Fig. 7 but for $200 \mathrm{MeV}$ nucleon energy.

descriptions of the target nucleus correlation function within the microscopic calculations of the second-order term of the KMT potential we show, in Fig. 10, the calculated nucleon- ${ }^{16} \mathrm{O}$ elastic scattering analyzing powers at $200 \mathrm{MeV}$. The curves shown are generated from the second-order optical potentials derived from the singleparticle (solid line), the CB (dot-dashed line), the Slater (dotted line), and the approximate (open circles) correlation functions. The corresponding curves for the differen-
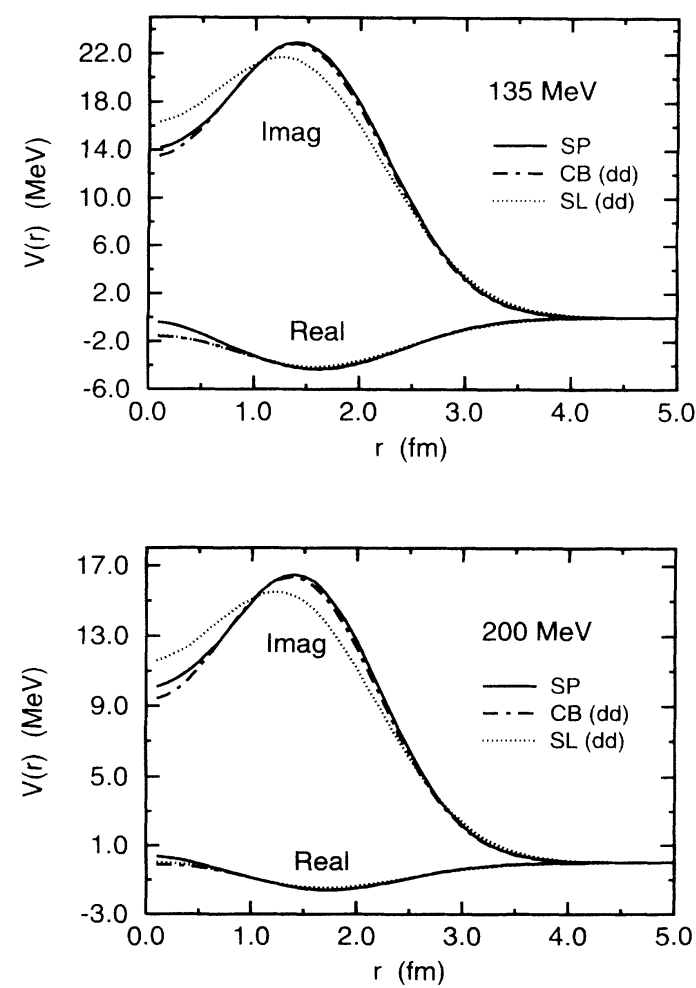

FIG. 9. Calculated zero range (of the $N N$ amplitude) limit of the second-order optical potential term derived from the single-particle correlation function (solid line), and the density dependent optical potentials derived from $\mathrm{CB}$ (dot-dashed line) and Slater (dotted line) correlation functions at 135 and $200 \mathrm{MeV}$. 


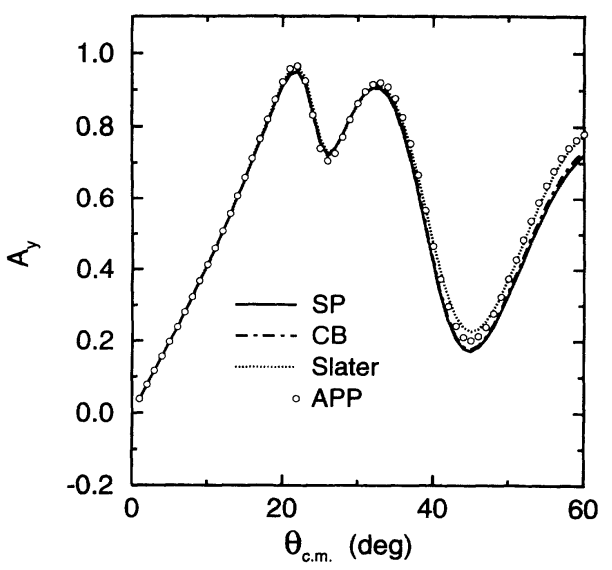

FIG. 10. Calculated nucleon- ${ }^{16} \mathrm{O}$ elastic scattering vector analyzing power at $200 \mathrm{MeV}$ using the optical potential derived from the single particle (solid line), the CB (dot-dashed line), the Slater (dotted line), and the approximate (app) (open circles) correlation functions.

tial cross sections show no variation and are not therefore presented. The figure shows that, within the microscopic calculations, the observables are rather insensitive to the details of the LDA to the correlation function.

Figures 11 and 12 on the other hand are used to compare the scattering observables calculated using the microscopic optical potential with those deduced from the nuclear matter limit through the LDA. Figure 11 shows the nucleon- ${ }^{16} \mathrm{O}$ elastic scattering differential cross sections calculated when using the microscopic (dot-dashed curve) and the approximate density-dependent (solid curve) second-order terms of the optical potential. Both calculations use the Campi-Bouyssy correlation function. Figure 12 shows the corresponding elastic vector analyzing powers derived using the microscopic potential, with CB (dot-dashed line) and Slater (dotted line) correlation

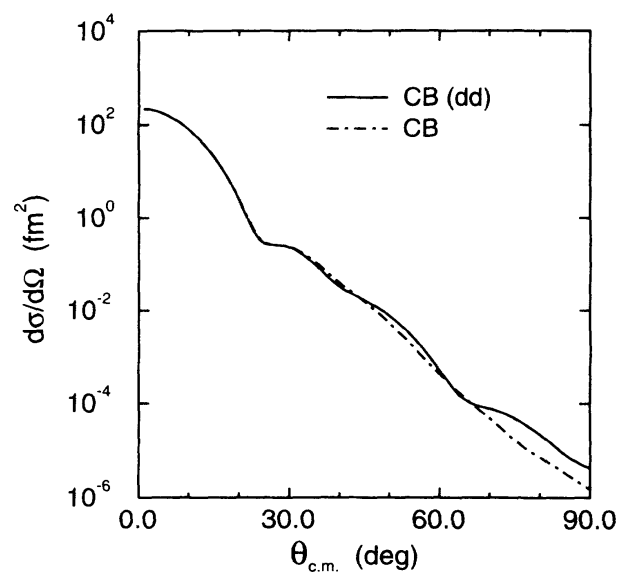

FIG. 11. Comparison of the nucleon- ${ }^{16} \mathrm{O}$ elastic scattering differential cross sections calculated using the microscopic (dot-dashed curve) and the density-dependent (DD) (solid curve) optical potentials derived using the $\mathrm{CB}$ correlation as described in the text.

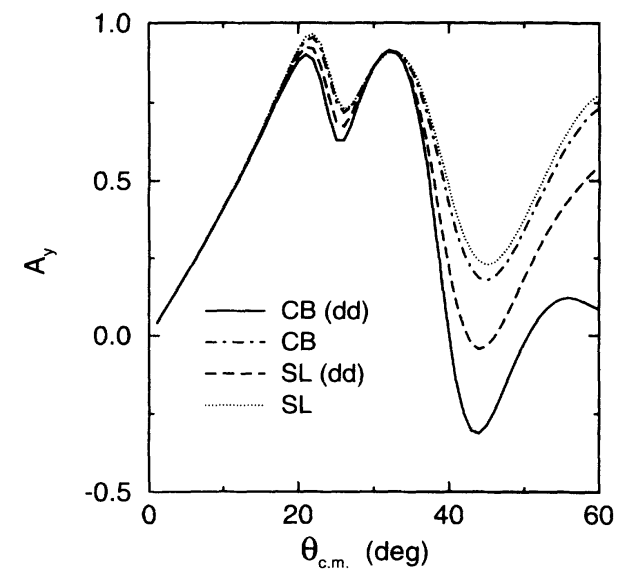

FIG. 12. Comparison of the nucleon- ${ }^{16} \mathrm{O}$ elastic scattering vector analyzing power calculated using the microscopic optical potentials derived from CB (dot-dashed line) and Slater (dotted line) correlation functions and the density-dependent (DD) optical potentials derived using the $\mathrm{CB}$ (solid line) and Slater (dashed line) correlations, as described in the text.

functions, and with the approximate density-dependent (DD) optical potential with CB (solid line) and Slater (dashed line) correlations. It is clear from these calculations that the use of the LDA for the Pauli blocking term of the microscopic optical potential, and which results in the surface peaking behavior in the second-order optical potential shown in Figs. 6 and 7, leads to changes in the optical potentials which have significant effects upon scattering calculations. This clearly has more general implications for other calculational methods which are based upon the use of the LDA.

\section{CONCLUSIONS}

This work presents a study of the validity of the local density approximation in calculations of the secondorder Pauli blocking term of the nucleon-nucleus interaction. Specifically, in order to clarify the effects of the use of the LDA upon the nuclear matter optical potential, we derive a WKB local equivalent potential representation to the second-order term of the Kerman-McManusThaler (KMT) multiple scattering expansion of the nucleon nucleus optical potential, following the formulation of Horiuchi [22]. To provide insight into the validity of local density approximations we use a simplified (Gaussian) parametrization of the required $N N$ form factors, as a function of the $N N$ energy and momentum transfer, and assume that the nucleon-nucleon transition amplitude can be calculated on the energy shell. In this way we are able to compare the microscopic KMT optical potential with the approximate optical potential deduced from the nuclear matter limit by use of the local density approximation. Calculations are presented for the nucleon- ${ }^{16} \mathrm{O}$ system at 135 and $200 \mathrm{MeV}$ incident energies for which earlier more exact calculations are also available for comparison. We have shown that calculated scattering observables are insensitive to the LDA treat- 
ment of the correlation function due to the fact that only the short range behavior, in the $N N$ separation distance, is sampled in the evaluation of the optical potential. Because to the finite range of the $N N$ transition amplitude, we show, however, that the derived local optical potential is very sensitive to approximations performed at the nuclear surface and inherent in the LDA prescriptions. It is shown that the use of the local density prescription leads to surface peaking of the optical potential, an effect which is absent from the second-order term of the optical potential derived microscopically from the KMT approach. The effects are significant and can be observed in calculations of nucleon elastic scattering observables.

We conclude that the local density approximation is a poor approximation to the treatment of Pauli block- ing effects included in microscopic calculations of the nucleon-nucleus optical potential. Care must be taken in the interpretation of the results obtained from formulations which make use of such local density prescriptions.

\section{ACKNOWLEDGMENTS}

The financial support of the United Kingdom Science and Engineering Research Council (SERC), now the Engineering and Physical Sciences Research Council (EPSRC), in the form of Grant Nos. GR/H53648 and GR/H53556, and No. GR/H89678 (for R.C.) are gratefully acknowledged.

\section{APPENDIX}

The Wigner transform of the second-order optical potential involves the evaluation of the folding of the product of the $N N$ amplitudes with the target correlation function, i.e.,

$$
F\left(\mathbf{r}, \mathbf{r}^{\prime}\right)=\int d \mathbf{r}_{1} d \mathbf{r}_{2} t_{01}^{f}\left(\omega, \mathbf{r}-\mathbf{r}_{1}\right) t_{01}^{f}\left(\omega, \mathbf{r}^{\prime}-\mathbf{r}_{2}\right) D\left(\mathbf{r}_{1}, \mathbf{r}_{2}\right)
$$

In the configuration space integrals, we replace

$$
t_{01}^{f}(\omega, s) t_{01}^{f}\left(\omega, s^{\prime}\right)=\hat{\beta}_{0}(\omega) \exp \left(-c\left[s^{2}+s^{\prime 2}\right]\right)
$$

where $\hat{\beta}_{0}(\omega)=[4 c \pi]^{3} \beta_{0}(\omega)$, with $\beta_{0}(\omega)=\beta(\omega, 0,0)$, given by Eq. $(60)$.

Defining $\hat{F}$ in terms of the $N N$ relative and center-of-mass coordinates,

$$
\hat{F}_{\lambda}(r, s)=\hat{\beta}_{0}(\omega) e^{-c\left[4 r^{2}+s^{2}\right] / 2} \int R_{12}^{2} d R_{12} \int s_{12}^{2} d s_{12} e^{-c\left[4 R_{12}^{2}+s_{12}^{2}\right] / 2} \mathcal{K}_{\lambda}\left(r, s, R_{12}, s_{12}\right)
$$

where

$$
\mathcal{K}_{\lambda}\left(r, s, R_{12}, s_{12}\right)=(4 \pi)^{2} D_{\lambda}\left(R_{12}, s_{12}\right) j_{\lambda}\left(i 4 c r R_{12}\right) j_{\lambda}\left(i c s s_{12}\right)
$$

These equations can be rewritten

$$
\hat{F}_{\lambda}(r, s)=\hat{\beta}_{0}(\omega) e^{-c\left[4 r^{2}+s^{2}\right] / 2} \int R_{12}^{2} d R_{12} \int s_{12}^{2} d s_{12} D_{\lambda}\left(R_{12}, s_{12}\right) \mathcal{R}_{\lambda}\left(r, s, R_{12}, s_{12}\right),
$$

where $\mathcal{R}_{\lambda}\left(r, s, R_{12}, s_{12}\right)$ is readily obtained from Eqs. (A3-A4) as

$$
\mathcal{R}_{\lambda}\left(r, s, R_{12}, s_{12}\right)=(4 \pi)^{2} e^{-c\left[4 R_{12}^{2}+s_{12}^{2}\right] / 2} j_{\lambda}\left(i 4 c r R_{12}\right) j_{\lambda}\left(i c s s_{12}\right) .
$$

We also consider the folding of the $N N$ scattering components with a density-dependent correlation function:

$$
F_{\rho}\left(\mathbf{r}-\mathbf{r}^{\prime}\right)=\int d \mathbf{r}_{1} d \mathbf{r}_{2} t_{01}^{f}\left(\omega, \mathbf{r}-\mathbf{r}_{1}\right) t_{01}^{f}\left(\omega, \mathbf{r}^{\prime}-\mathbf{r}_{2}\right) D\left(\rho,\left|\mathbf{r}_{1}-\mathbf{r}_{2}\right|\right)
$$

Upon performing the integrals, and applying the $\operatorname{LDA}, \rho \rightarrow \rho(r), \hat{F}_{\rho}(s) \rightarrow \hat{F}^{\mathrm{LDA}}(r, s)$, then

$$
\hat{F}^{\mathrm{LDA}}(r, s)=\hat{\beta}_{0}(\omega) e^{-c s^{2} / 2} \int d s_{12} s_{12}^{2} e^{-c s_{12}^{2} / 2}(4 \pi)\left(\frac{\pi}{2 c}\right)^{3 / 2} j_{0}\left(i c s s_{12}\right) D\left(\rho(r), s_{12}\right)
$$

or

$$
\hat{F}^{\mathrm{LDA}}(r, s)=\hat{\beta}_{0}(\omega) e^{-c s^{2} / 2} \int d s_{12} s_{12}^{2} \mathcal{R}^{\mathrm{LDA}}\left(s, s_{12}\right) D\left(\rho(r), s_{12}\right)
$$

with

$$
\mathcal{R}^{\mathrm{LDA}}\left(s, s_{12}\right)=(4 \pi)\left(\frac{\pi}{2 c}\right)^{3 / 2} e^{-c s_{12}^{2} / 2} j_{0}\left(i c s s_{12}\right)
$$


[1] A.K. Kerman, H. McManus, and R.M. Thaler, Ann. Phys. (N.Y.) 8, 551 (1959).

[2] G. Takeda and K.M. Watson, Phys. Rev. 97, 1336 (1955).

[3] J. Hüfner and C. Mahaux, Ann. Phys. (N.Y.) 73, 299 (1972).

[4] F.A. Brieva and J.R. Rook, Nucl. Phys. A281, 317 (1972); H. von Geramb, in Microscopic Optical Potentials, edited by H. von Geramb (Springler-Verlag, Berlin, 1987), p. 104.

[5] J.P. Jeukenne, A. Lejeune, and C. Mahaux, Phys. Rep. C 25, 83 (1976).

[6] C. Mahaux, Nucl. Phys. A396, 9c (1983).

[7] L. Rikus, K. Nakano, and H. von Geramb, Nucl. Phys. A414, 413 (1984).

[8] R. Crespo, R.C. Johnson, and J.A. Tostevin, Phys. Rev. C 44, R1735 (1991); 46, 279 (1992).

[9] R.C. Johnson and D.C. Martin, Nucl. Phys. A192, 496 (1972); A211, 617 (1973).

[10] H. Feshbach and J. Hüfner, Ann. Phys. (N.Y.) 56, 268 (1970); H. Feshbach, A. Gal, and J. Hüfner, ibid. 66, 20 (1971).

[11] E.F. Redish and K. Stricker-Bauer, Phys. Rev. C 36, 513 (1987).

[12] M. Lacombe, B. Loiseau, J.M. Richard, R. Vinh Mau, J. Côté, P. Pires, and R. de Tourreil, Phys. Rev. C 21, 861 (1980).

[13] For a complete review, see R. Machleidt, in Advances in Nuclear Physics, edited by J.W. Negele and E. Vogt
(Plenum Press, New York, 1989), Vol. 19, Chap. 2.

[14] H.F. Arellano, F.A. Brieva, and W.G. Love, Phys. Rev. C 41, 2188 (1990); H.F. Arellano, W.G. Love, and F.A. Brieva, ibid. 43, 2734 (1991).

[15] Ch. Elster, T. Cheon, E.F. Redish, and P.C. Tandy, Phys. Rev. C 41, 814 (1990); C.R. Chinn, Ch. Elster, and R.M. Thaler, ibid. 44, 1569 (1991).

[16] R. Crespo, R.C. Johnson, and J.A. Tostevin, Phys. Rev. C 41, 2257 (1990).

[17] R. Crespo, R.C. Johnson, and J.A. Tostevin, Phys. Rev. C 48, 351 (1993).

[18] C.R. Chinn, Ch. Elster, and R.M. Thaler, Phys. Rev. C 48, 2956 (1993).

[19] R. Crespo, R.C. Johnson, J.A. Tostevin, R.S. Mackintosh, and S.G. Cooper, Phys. Rev. C 49, 1091 (1994).

[20] M. Kleinmann, R. Fritz, and H. Müther, report, 1994.

[21] P. Czerski, H. Müther, and W.H. Dickoff, J. Phys. G 20, 425 (1994).

[22] H. Horiuchi, Prog. Theor. Phys. 64, 184 (1980).

[23] V.R. Pandharipande, Nucl. Phys. A135, 419 (1969).

[24] J.W. Negele and D. Vautherin, Phys. Rev. C 11, 1031 (1975).

[25] X. Campi and A. Bouyssy, Phys. Lett. 73B, 263 (1978).

[26] An extension of the computer programs of E.F. Redish (unpublished) to calculate all of the KMT $N N$ scattering amplitudes.

[27] T.W. Donnelly and G.E. Walker, Phys. Rev. Lett. 22, 1121 (1969). 